\title{
Effectiveness of Helmet Cranial Remodeling in Older Infants with Positional Plagiocephaly
}

Hong Youl Kim, Yoon Kyu Chung, Yong Oock Kim

Department of Plastic and Reconstructive Surgery, Institute for Human Tissue Restoration, Yonsei University College of Medicine, Seoul, Korea
No potential conflict of interest relevant to this article was reported.

\begin{abstract}
Background: Management of positional plagiocephaly by wearing a cranial molding helmet has become a matter of growing medical interest. Some research studies reported that starting helmet therapy early (age 5 to 6 months) is important and leads to a significantly better outcome in a shorter treatment time. The aim of the present study was to evaluate the effectiveness of cranial remodeling treatment with wearing helmet for older infants ( $\geq 18$ months).

Methods: We conducted a retrospective study of 27 infants with positional plagiocephaly without synostosis, who were started from 2008 to 2012. Every child underwent a computerized tomography (CT) before starting helmet therapy to exclude synostosis of the cranial sutures and had CT performed once again after satisfactory completion of therapy. Anthropometric measurements were taken on using spreading calipers in every child. The treatment effect was compared using cranial vault asymmetry (CVA) and the cranial vault asymmetry index (CVAl), which were obtained from diagonal measurements before and after therapy.

Results: The discrepancy of CVA and CVAI of all the patients significantly decreased after cranial molding helmet treatment in older infants ( $\geq 18$ months) $7.6 \mathrm{~mm}$ from 15.6 $\mathrm{mm}$ to $8 \mathrm{~mm}$ and $4.51 \%$ from $9.42 \%$ to $4.91 \%$. Six patients had confirmed successful outcome, and all subjects were good compliance patients. The treatment lasted an average of 16.4 months, was well tolerated, and had no complication. Additionally, the rate of the successful treatment (final CVA $\leq 5 \mathrm{~mm}$ ) significantly decreased when the wearing time per was shorter.

Conclusion: This study showed that treatment by cranial remodeling orthosis was effective if the patient could wear the helmet longer and treatment duration was somewhat longer than in younger patients, well tolerated in older infants and had no morbidity. This therapeutic option is available and indicated in these older infants before other cranial remodeling surgery.
\end{abstract}

Keywords: Positional plagiocephaly / Helmet therapy / Cranial vault asymmetry / Cranial vault asymmetry index

\section{INTRODUCTION}

Positional plagiocephaly is a condition of asymmetrical cranial morphology caused by an extrinsic factor, such as pressure, rather

Correspondence: Yong Oock Kim

Department of Plastic and Reconstructive Surgery, Gangnam Severance Hospital, Yonsei University College of Medicine, 211 Eonju-ro, Gangnam-gu, Seoul 135-720, Korea

E-mail: sgm625@yuhs.ac

Received June 30, 2014 / Revised July 29, 2014 / Accepted August 5, 2014 than an intrinsic factor [1]. It is also called non-synostotic plagiocephaly or deformational plagiocephaly. There are many causes of positional plagiocephaly, but sleeping position is assumed to play a major role in etiology. The incidence of positional plagiocephaly has increased with the start of 'Back to Sleep' campaign by the American Academy of Pediatrics [2-6] and ranges from 1 in 300 live births to 22.1 percent $[7,8]$.

Increasing quality of life and awareness of problems caused by positional plagiocephaly have raised parental demand for correc- 
tion of this condition. In recent years, the number of parents seeking counsel for positional plagiocephaly has increased at our clinic.

Many studies that have reported that positional plagiocephaly does not improve naturally [9]. Asymmetry of face and position of ear can remain permanent [1]. Helmet orthoses can restore a degree of symmetry to the cranium by limiting growth in protruded areas and by allowing rapid growth in areas which are flat [10].

Until now, studies have indicated that early initiation of helmet therapy is important and leads to a significantly better outcome within a shorter treatment time. However, few studies have reported helmet therapy in older patients. The purpose of this study was to determine whether helmet therapy was effective in older children and to further identify factors associated with desired outcomes.

\section{METHODS}

A retrospective study was conducted for and identified 27 infants with positional plagiocephaly between 2008 and 2012. The inclusion criteria of this study were 1) diagnosis of non-synostotic plagiocephaly without surgical treatment, 2) over 18 months of age when cranial remodeling helmet was begun, and 3) completion of helmet therapy or regular follow-up for at least one year with complete documentation.

Every child included in the study underwent a computerized tomography (CT) before starting helmet therapy to exclude synostoses of the cranial sutures. A repeat CT scan was performed after completion of therapy (Fig. 1).

Anthropometric measurements were made using calipers, as well as using CT images. Effectiveness of treatment was compared using cranial vault asymmetry (CVA) and the cranial vault asymmetry index (CVAI), which were obtained from measurements taken before and after therapy (Fig. 2).

The most prominent frontozygomatic area-to-contralateral occipital distance was measured on both sides in every patient, using a spreading caliper. The $\mathrm{f}_{\mathrm{A}}$ is the most prominent frontozygomatic area and euA is the oppose side from fza. Thus the longest distance in the vertex view is form $\mathrm{fZA}_{\mathrm{A}}$ to euA. The fZв means the most depressed frontozygomatic area and еuв is other side from fzв. The distances were defined as diagonal A (the distance from $\mathrm{fZA}_{\mathrm{A}}$ to $\mathrm{eu}_{\mathrm{A}}$ ) or $\mathrm{B}$ (the distance from $\mathrm{fz}_{\mathrm{B}}$ to $\left.\mathrm{eu}_{\mathrm{B}}\right)(\mathrm{A}>\mathrm{B})$, and the cranial vault asymmetry (CVA) (diagonal A-diagonal B) and cranial vault asymmetry index (CVAI) ([diagonal A-diagonal B]/[diagonal A× 100]) [10] were measured. CVA and CVAI were compared from the initial measurement to measurements made at each visit to assess the treatment progress (Fig. 1). A final CVA less than $5 \mathrm{~mm}$ was considered as an indicator of successful treatment.

We also analyzed patient compliance. Good compliance meant helmet application for greater than 17 hours per a day. All patients with successful outcomes wore the helmet for greater than 17 hours.

Patients were divided into successful and unsuccessful out-
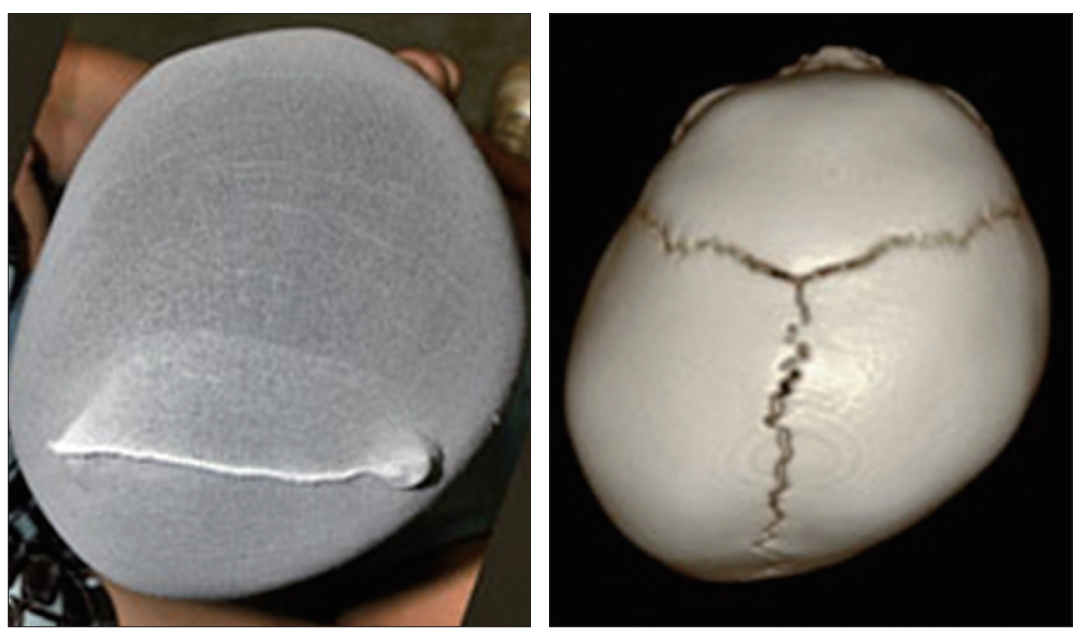

Fig. 1. Appearance and computed tomography of a positional plagiocephaly patient. 

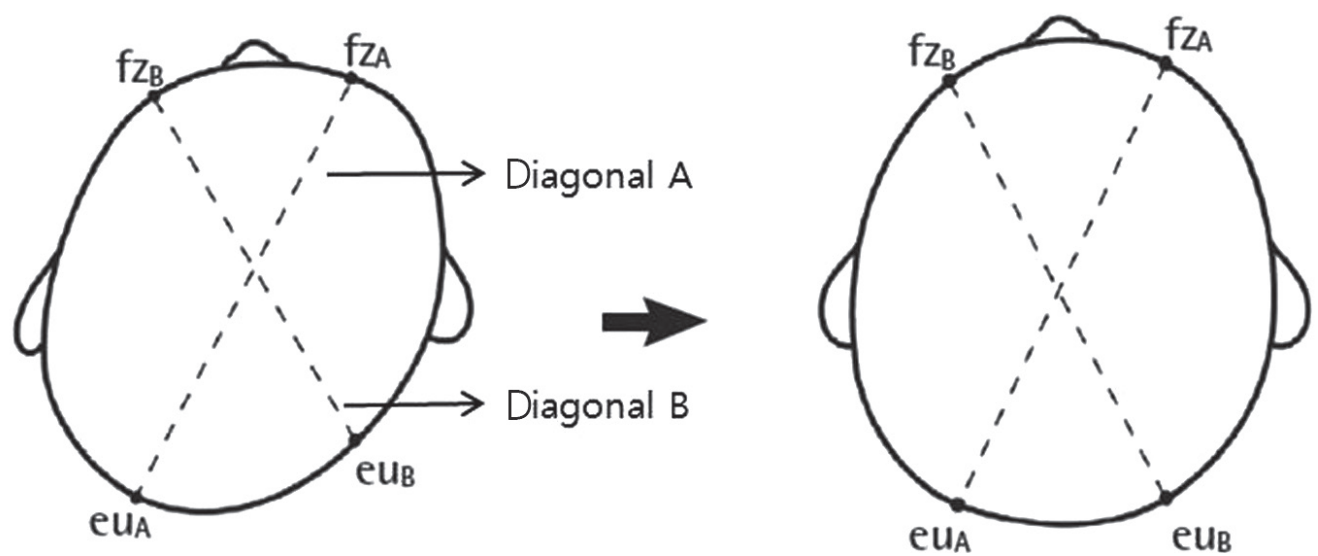

Fig. 2. Diagonal measurement of patient and the treatment effect of the helmet therapy strategy.

come groups, and were compared using a paired sample T-test and one way analysis of variance test. Differences were considered statistically significant for $p<0.05$. Data analysis was performed using SPSSWIN ver. 21.0. (IBM Co., Armonk, NY, USA).

\section{RESULTS}

Of the 27 patients, the mean age was 29.7 months (range, 18 to 75 months) at initiation of helmet therapy. The initial mean CVA and

Table 1. The patient's initial value, compliance and helmet therapy duration

\begin{tabular}{lcc} 
Variable & Value & Range \\
\hline Initial CVA (mm) & 15.6 & $6-26$ \\
\hline Initial CVAl (\%) & 9.42 & $3.53-16.05$ \\
\hline Compliance (hr/day) & 17.5 & $10-22.3$ \\
Helmet therapy duration (mo) & 16.4 & $7-33.6$ \\
\hline
\end{tabular}

CVA, cranial vault asymmetry; CAVI, cranial vault asymmetry index.
CVAI were $15.6 \mathrm{~mm}$ (range, 6 to $26 \mathrm{~mm}$ ) and 9.42\% (range, 3.53\% to $16.08 \%$ ), respectively. The mean helmet application time was 17.5 hours per a day (range, 10 to 22.3 hours), and the mean duration of overall treatment was 16.4 months (range, 7 to 33.6 months) (Table 1).

At the end of follow up period, the mean CVA and CVAI were $8 \mathrm{~mm}$ (range, 1 to $15 \mathrm{~mm}$ ) and $4.91 \%$ (range, $0.59 \%$ to $9.68 \%$ ). This was approximately a $50 \%$ improvement compared to the initial CVA and CVAI, respectively $(p<0.001)$.

Six of 27 patients (22\%) were considered to have successful outcomes. For this group of 6 patients, the mean age at helmet therapy initiation was 27.2 months, with initial CVA of $12.5 \mathrm{~mm}$ versus final CVA of $2.5 \mathrm{~mm}$. The mean compliance was 21.2 hour/day (Table 2). Interestingly, the mean helmet therapy duration was shorter for patients with successful outcomes than those without (10.8 months vs. 18.4 months). Initial CVA, CVAI, or age at initiation of therapy were not associated with successful outcomes.

Table 2. The result of successful outcome patient

\begin{tabular}{lccccccc} 
No. & $\begin{array}{c}\text { Age of start } \\
\text { helmet therapy }(\mathrm{mo})\end{array}$ & $\begin{array}{c}\text { Initial CVA } \\
(\mathrm{mm})\end{array}$ & $\begin{array}{c}\text { Final CVA } \\
(\mathrm{mm})\end{array}$ & $\begin{array}{c}\text { Initial CVAl } \\
(\%)\end{array}$ & $\begin{array}{c}\text { Final CVAl } \\
(\%)\end{array}$ & $\begin{array}{c}\text { Treatment period } \\
(\mathrm{mo})\end{array}$ & $\begin{array}{c}\text { Compliance } \\
(\mathrm{hr} / \mathrm{day})\end{array}$ \\
\hline 1 & 31.3 & 9.0 & 1.0 & 5.36 & 0.61 & 12.2 & 21.3 \\
\hline 2 & 17.1 & 12.0 & 1.0 & 7.14 & 0.59 & 7.8 & 19.2 \\
\hline 3 & 26.9 & 22.0 & 13.0 & 4.07 & 1.18 & 5.9 & 19.6 \\
\hline 4 & 33.7 & 7.0 & 2.0 & 2.94 & 1.78 & 2.4 & 13.4 \\
\hline 5 & 28.8 & 19.0 & 4.0 & 11.18 & 2.48 & 19.4 & 21.0 \\
\hline 6 & 25.6 & 6.0 & 4.0 & 3.53 & 2.38 & 12.2 & 20.3 \\
\hline Mean & 27.2 & 12.5 & 2.5 & 6.56 & 1.51 & 10.84 & 20.2 \\
\hline
\end{tabular}

CVA, cranial vault asymmetry; CAVI, cranial vault asymmetry index. 
However, final CVA, final CVAI, treatment duration, and daily helmet wear were significantly different (Table 3).

Those who were confirmed as poor compliance among patients with failed treatment $(\mathrm{n}=6)$ had improved CVA by $5 \mathrm{~mm}$ only (from 16 to $11 \mathrm{~mm}$ ) and CVAI was 2.96\% (from 9.23\% to $6.27 \%$ ), even though their mean treatment period was comparatively long at 20.6 months. Patients with good compliance but with treatment failure $(\mathrm{n}=12)$ had improved CVA and CVAI from 17 to $10 \mathrm{~mm}$ and $10.63 \%$ to $5.28 \%$, respectively. Despite not being determined as a successful outcome, their results were considerably better (Table 3 ).

There were no complications during the follow-up period.

\section{Case}

A male toddler was begun on helmet treatment at 23 months of age. The child wore the helmet for an average of 22.3 hours a day with an overall treatment duration of 6.3 months (Fig. 3). His initial CVA and final CVA were $19 \mathrm{~mm}$ and $4 \mathrm{~mm}$, respectively. Initial CVAI was $11.18 \%$, and final CVAI was $2.48 \%$.

\section{DISCUSSION}

In the past two decades, parents have shown increasing interest in positional plagiocephaly. Awareness regarding plagiocephaly and the resultant asymmetry of face and unforeseen functional prob-

Table 3. The result of patient data about compliance

\begin{tabular}{|c|c|c|c|c|c|}
\hline \multirow{2}{*}{ Variable } & \multirow{2}{*}{$\frac{\text { Success }}{\text { Good compliance }(n=6)}$} & \multicolumn{3}{|c|}{ Fail } & \multirow{2}{*}{$p$-value } \\
\hline & & Good compliance $(n=12)$ & Poor compliance $(n=9)$ & Mean & \\
\hline Age of start helmet therapy (mo) & 27.2 & 31.9 & 28.3 & 30.3 & 0.232 \\
\hline Initial CVA (mm) & 12.5 & 17 & 16 & 17 & 0.216 \\
\hline Final CVA (mm) & 2.5 & 10 & 11 & 9 & 0.000 \\
\hline Initial CVAI (\%) & 6.56 & 10.63 & 9.23 & 10.03 & 0.168 \\
\hline Final CVAI (\%) & 1.51 & 5.28 & 6.27 & 5.71 & 0.000 \\
\hline Treatment period (mo) & 10.84 & 17.53 & 18.31 & 18.42 & 0.001 \\
\hline Compliance (hr/day) & 21.2 & 18.9 & 14.9 & 17.1 & 0.000 \\
\hline
\end{tabular}

CVA, cranial vault asymmetry; CAVI, cranial vault asymmetry index.
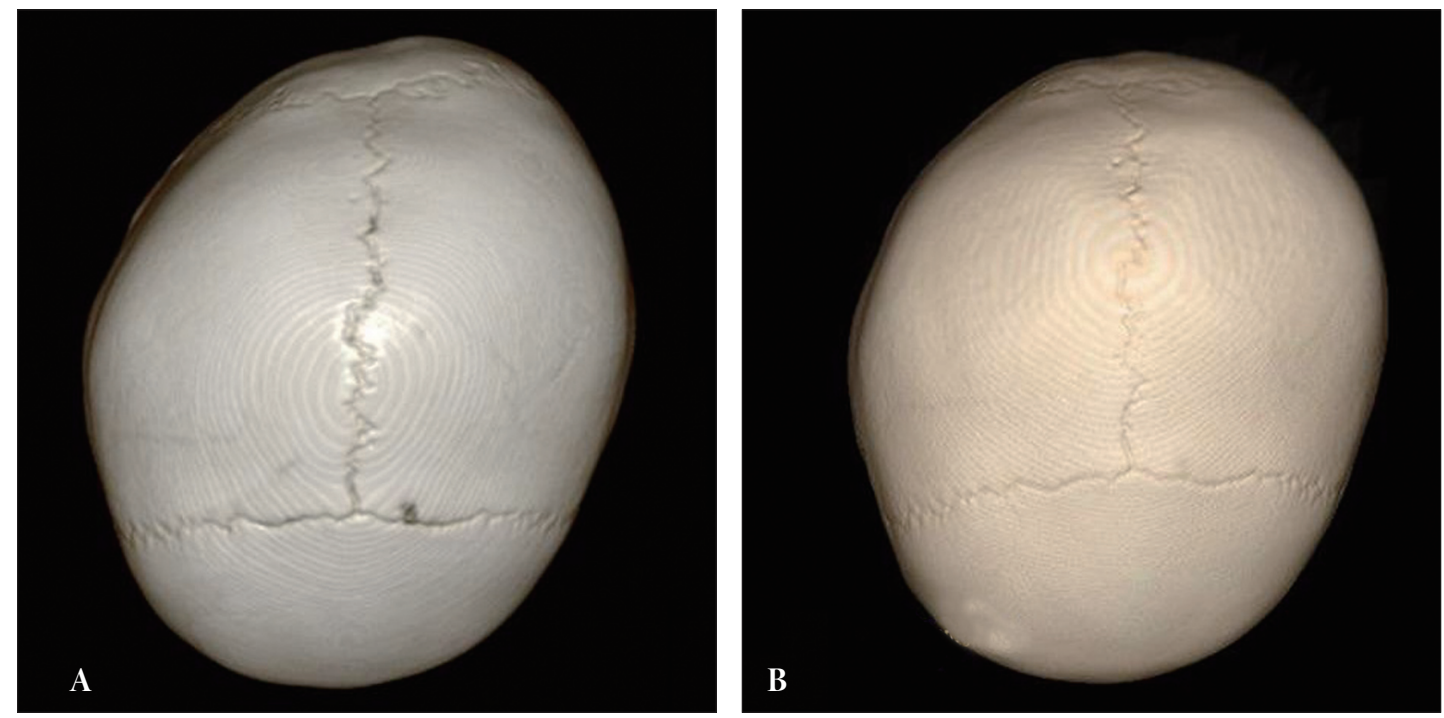

Fig. 3. Case. (A) Three dimensional (3D) computed tomography (CT) scan before helmet therapy, CVA=19 mm, CVAI=11.18\%. (B) 3D CT scan after 6.3 months helmet therapy, $\mathrm{CVA}=4 \mathrm{~mm}, \mathrm{CVAI}=2.48 \%$. CVA, cranial vault asymmetry; $\mathrm{CAVI}$, cranial vault asymmetry index. 
lems has led to a rising demand for treatment [11]. A number of articles have addressed this issue. There are several possible treatment options, such as physiotherapy, head repositioning, and helmet therapy $[12,13]$. Most studies have reported that treatment such as physiotherapy and head repositioning fail after first 6 month of life [13].

While the timing of helmet therapy is considered important, also, the debate continues regarding the optimal timing of helmet therapy as the most important factor influencing the outcome. The current consensus is that helmet treatment is best if initiated within the first 12 months of life, since $85 \%$ of cranial growth occurs during this time. However, helmet orthoses are considered effective up to 18 months of age. Grigsby [14] compared differences in treatment duration and outcome of cranial molding therapy between children younger and older than 12 months and 12 months. The authors found that cranial molding treatment was effective in the older group, but required twice the treatment duration for similar amount of correction.

It is no uncommon that parents visit our out-patient clinic with children who are older than 18 months with significant positional plagiocephaly. In the past, we recommend against helmet therapy, considering the lack of evidence in these older children and the high cost of these custom helmets. Fully aware of these factors, however, some parents expressed strong desire to proceed with helmet treatement for their child. We therefore became interested in determining whether this treatment modality was still effective in these older children.

Among the children studied, the mean CVA and CVAI had improved from 15.6 to $8 \mathrm{~mm}$ and from $9.42 \%$ to $4.91 \%$, respectively. Both of these before-after differences were statistically significant. These results indicated that head shape could be improved in infants older than 18 months, and also that helmet therapy was effective in these older children. The average helmet therapy duration of 16.4 months was higher than those reported in other studies. Kluba et al. [15] reported the time to successful outcome in patients younger than 6 months and older than 6 months were 14 weeks and 18 weeks of treatment, respectively. In their study, patients younger than 6 months had CVA improvement from 17 to $4.5 \mathrm{~mm}$, and CVAI was from $13.6 \%$ to $3.1 \%$. Patients older than 6 months had CVA improvement from 17 to $6.5 \mathrm{~mm}$, and CVAI was from $13.1 \%$ to $4.5 \%$.

Six of 27 patients were considered to have successful treatment outcome. All wore the helmet for more than 17 hours per day, and the mean helmet wearing time was 21.2 hours per day. Their CVA was improved, $10 \mathrm{~mm}$ (from 12.5 to $2.5 \mathrm{~mm}$ ) and CVAI was $5.05 \%$ (from $6.56 \%$ to $1.51 \%$ ). The treatment duration for this subgroup was 10.8 months.

Among patients who wore the helmet for less than 17 hours per a day, the CVA and CVAI was improved only $5 \mathrm{~mm}$ (from 16 to 11 $\mathrm{mm}$ ) and $2.96 \%$ (from $9.23 \%$ to $6.27 \%$ ), respectively. Their treatment period was 20.6 months (Table 3). In a comparison between successful and unsuccessful outcome groups, there was no significant difference in the initial CVA, initial CVAI, and age at helmet treatment initiation. However, final CVA, final CVAI, compliance, treatment period, and compliance were significantly different. It means that good compliance could be a key of successful outcome, and can shortening helmet wearing duration. These results indicate that a more consistent sue of the helmet (maximal hours each day) can achieve a successful outcome, and can shorten the overall duration of treatment. Twelve patients were considered to be in good compliance with a mean of 18.9 hours per day but did not reach successful outcome. Their CVA and CVAI were improved by a difference of $7 \mathrm{~mm}$ and $5.35 \%$, respectively. These values represent relative improvements of approximately $50 \%$. The helmet treatment was effective in infants older than 18 months.

The limitation of our study was that we included completely treated patients. There was selection bias in this study because patients with better outcomes had no complications and more easily completed the treatment and came back for follow-up visits. Because of the small study size, we could not suggest an optimal duration for helmet treatment for this older population of infants. If we are able to obtain a larger sample, we will conduct a future study to answer this question. Furthermore, long-term follow up is needed to examine psychological aspects of using these devices for children who are beginning the social phases of their development.

This report reached a definitive conclusion that helmet treatment was effective in infants older than 18 months whose parents are able to comply with a strict helmet wearing regimen. Compli- 
ance was crucial to successful outcome; otherwise, longer treatment durations were needed for older patients and was associated with additional burdens like cleansing, checking for correct fitness, and skin irritation. However, if the helmet was used for more than 20 hours per a day, the duration of treatment was somewhat shortened.

\section{REFERENCES}

1. Kelly KM, Littlefield TR, Pomatto JK, Manwaring KH, Beals SP. Cranial growth unrestricted during treatment of deformational plagiocephaly. Pediatr Neurosurg 1999;30:193-9.

2. American Academy of Pediatrics AAP Task Force on infant positioning and SIDS: positioning and SIDS. Pediatrics 1992;89:1120-6.

3. Argenta LC, David LR, Wilson JA, Bell WO. An increase in infant cranial deformity with supine sleeping position. J Craniofac Surg 1996;7:5-11.

4. Kane AA, Mitchell LE, Craven KP, Marsh JL. Observations on a recent increase in plagiocephaly without synostosis. Pediatrics 1996;97:877-85.

5. Turk AE, McCarthy JG, Thorne CH, Wisoff JH. The "back to sleep campaign" and deformational plagiocephaly: is there cause for concern? J Craniofac Surg 1996;7:12-8.

6. McKinney CM, Cunningham ML, Holt VL, Leroux B, Starr JR. Characteristics of 2733 cases diagnosed with deformational plagio- cephaly and changes in risk factors over time. Cleft Palate Craniofac J 2008;45:208-16.

7. van Vlimmeren LA, van der Graaf Y, Boere-Boonekamp MM, L'Hoir MP, Helders PJ, Engelbert RH. Risk factors for deformational plagiocephaly at birth and at 7 weeks of age: a prospective cohort study. Pediatrics 2007;119:e408-18.

8. Dunn PM. Congenital sternomastoid torticollis: An intrauterine postural deformity. Arch Dis Child 1974;49:824-5.

9. Boere-Boonekamp MM, van der Linden-Kuiper LL. Positional preference: prevalence in infants and follow-up after two years. Pediatrics 2001;107:339-43.

10. Schaaf H, Malik CY, Streckbein P, Pons-Kuehnemann J, Howaldt HP, Wilbrand JF. Three-dimensional photographic analysis of outcome after helmet treatment of a nonsynostotic cranial deformity. J Craniofac Surg 2010;21:1677-82.

11. Kluba S, Schreiber R, Kraut W, Meisner C, Reinert S, Krimmel M. Does helmet therapy influence the ear shift in positional plagiocephaly? J Craniofac Surg 2012;23:1301-5.

12. Morrison CS, Chariker M. Positional plagiocephaly: pathogenesis, diagnosis, and management. J Ky Med Assoc 2006;104:136-40.

13. Robinson S, Proctor M. Diagnosis and management of deformational plagiocephaly. J Neurosurg Pediatr 2009;3:284-95.

14. Grigsby K. Cranial remolding helmet treatment of plagiocephaly: comparison of results and treatment length in younger versus older infant populations. J Prosthet Orthot 2009;21:55-63.

15. Kluba S, Kraut W, Reinert S, Krimmel M. What is the optimal time to start helmet therapy in positional plagiocephaly? Plast Reconstr Surg 2011;128:492-8. 